\title{
Classification of RSS-Formatted Documents Using Full Text Similarity Measures
}

\author{
Katarzyna Wegrzyn-Wolska ${ }^{1}$ and Piotr S. Szczepaniak ${ }^{2,3}$ \\ 1 Ecole Supérieure d'Ingenieurs en Informatique et Génie de Télécommunication \\ 77-200 Avon-Fontainebleau, France \\ 2 Institute of Computer Science, Technical University of Lodz, \\ ul. Wolczanska 215, 93-005 Lodz, Poland \\ 3 Systems Research Institute, Polish Academy of Sciences \\ ul. Newelska 6, 01-447 Warsaw, Poland
}

\begin{abstract}
The Web is enormous, unlimited and dynamically changed source of useful and varied kinds of information. The news is one of the most rapidly changing kinds of information. The departure for this paper is presentation of RSS, an useful data format used frequently by publishers of news; some statistics related to news syndication illustrate the actual situation. Then, two recently developed methods for examination of similarity of textual documents are briefly presented. Since RSS-supplied records always contain the same type of information (headlines, links, article summaries, etc.), application of methods of presented type makes their diverse applications like automatic news classification and filtering easier.
\end{abstract}

\section{Introduction}

This paper describes some general problems of publishing, retrieving and filtering news information on the Web. The first section introduces the principle of the news publication on the Web. The second section explains the main problems of exploration and filtering of the news data from the presentation Web pages. The following sections present the problems of the contents syndication on the Web and present some statistical results of the experiments carried out. Then the RSS feed data formats is introduced. The following sections show potential and features of this format. The last sections present methods for classification of documents using full text similarity measures. The conclusion summarizes the problems presented.

\section{News Published on the Web}

There are a lot of web sites which publish news. The news sites publish different kinds of information in different presentation forms [3, 6]. News is a very dynamic kind of information, constantly updated. The news sites have to be verified very often so as not to miss any of the news information. In Table 1 the updating frequency for some Web news sites is presented.Some parameters are significant

D. Lowe and M. Gaedke (Eds.): ICWE 2005, LNCS 3579, pp. 400-405, 2005.

(C) Springer-Verlag Berlin Heidelberg 2005 
Table 1. Example of updating news frequency (provided by the sites administrators)

\begin{tabular}{|c|l|c|}
\hline Service news & URL & Update \\
\hline \hline Google & http://news.google.com & about 20 min \\
\hline Voila actuality & http://actu.voila.fr & every day \\
\hline TF1 news & http://new.tf1.fr/news & instantaneously \\
\hline News now & http://ww.newsnow.co.uk & about 5 min \\
\hline CategoryNet & http://www.categorynet.com & every day \\
\hline CNN & http://www.cnn.com & instantaneously \\
\hline Company news groups & http://www.companynewsgroup.com & about 40 per day \\
\hline
\end{tabular}

for automatic treatment; frequency of data updating, the size of the transferred data and extraction and filtering facilities on news sites. We have done some statistical tests to evaluate the updating frequency [13] of news.

The results shown (Fig.1a) the different behavior of interrogated sites: the news updated very regular or irregular when information is updated when present. Some news sites present periodic activity: ex. the news site of the French television channel TF1 is updated only during working hours. The results confirm that the content of the news sites change very often. This is one of the most important reasons for careful optimization the data flux format. The traffic generated on the net by news is high and it is interesting to optimize it. We have done some comparative tests (Fig. 1b) of the transferred data size for the news presented in HTML and in XML (RSS).

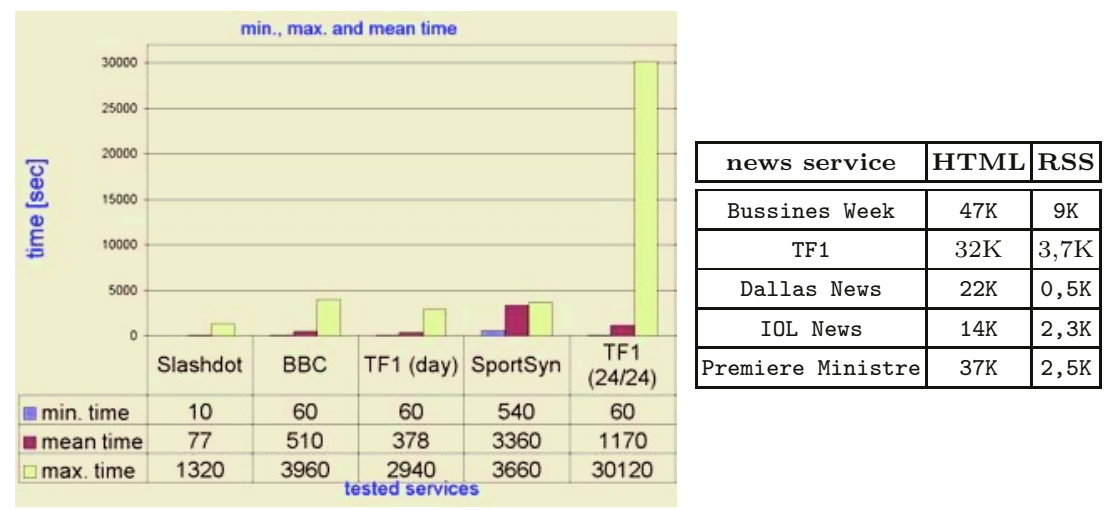

Fig. 1. a) Values of updating frequency; b) comparative size of HTML and RSS

\section{News Data Formats: HTML and RSS}

There are two the most frequently used data format for news publishing; the HTML non-structured data format and the dedicated format, named RSS-feed. These formats have different aspects for publication and presentation and for retrieving and exploring the data by other tools such as catalogues, searchengines and meta-search tools. 


\subsection{HTML Presentation}

There are a lot of news Web sites that present the news using only the standard HTML page form. This page contains a lot of diverse data, not only lists of selected news items, but also much additional information. This additional information is completely useless for the retrieving tools (Search Engine, MetaSearch Engine, etc.) [11, 12]. The searching news' agent needs to extract only the significant data. Additional and non-essential data increases the complexity of analysis. The HTML pages, which include the informational and presentational data, are not optimal for data extraction and information content updating. The HTML pages are also not optimal for data transfer because of their size.

Data Extraction Problems. The format of the HTML news pages are not standardized. Their form does not lend itself to information extraction. There are two kinds of extraction problems. Firstly, finding all of the news description with their links included in the news page and then identifying only the pertinent ones. The most important difficulties of information extraction are: complex linking, difficulties in recognizing and following links to framed pages and then extraction of the information in this frame, difficulties in identifying links in image maps and in the script code sources like a JavaScript, etc. There is also some information, which is not static, for example, thematic publicity selected automatically and frequently changed. The retrieving tool has to distinguish which piece of data is the news information and select only the significant links.

\subsection{RSS Feed}

RSS (RDF Site Summary) is an XML-based special format that enables web developers to describe and syndicate web site content. The original RSS, was designed as a format for building portals of headlines to mainstream news sites. But RSS is not designed just for news and news-like sites, it became one of the basic weblog-oriented products and other web-based publishing software.

RSS Advantages. RSS provides a static and well-structured format for all the textual documents. The RSS file contains only the informational data formatted in a standardized format without any presentation parts. This well structured document is easy to parse. It is possible to analyze, monitor and to extract the data automatically. When we aim at off-line filtering or classification of news or other documents the RSS format seems to be a good proposition for simple extracting textual records which needs to be evaluated on their relevance. In the evaluation, human-similar comparison of textual documents (words, sentences, abstracts, full documents, etc.) is a key problem.

\section{Similarity of Textual Records}

So far, the methods used for text comparison have been based mainly on the classical identity relation, according to which two given texts are either identical 
or not. Diverse similarity or distance measures for sequence of characters have been developed. Examples of simple indices are Hamming and Levenstein distances. However, conventional methods are of limited usefulness and reliability, in particular for languages having reach inflexion (e.g.Slavonic languages). Here, two more sophisticated methods that make use of different independent ways of looking for similarity - similarity in terms of fuzzy sets theory [7, 9, 10] and sequence kernels [2, 4, 5], are proposed as possible solution. Although they exhibit certain similarities in their behavior, in many aspects the methods differ in an essential way. Here, only the first method is briefly presented; for the second one we refer to the given literature.

Fuzzy Measure. To enable a computer compare textual documents, the fuzzy similarity measure proposed in [7] (cf. also [9, 10]) can be used. Because in the considered case we deal with relatively frequently changing sources of textual information, the less time-consuming version of the method [8] analogous to the $n$-gram method described in [1] is recommended.

Let $W$ be the set of all words from a considered dictionary (universe of discourse).

Definition 1. The similarity measure takes the form

$$
\forall w_{1}, w_{2} \in W: \quad \mu_{R W}\left(w_{1}, w_{2}\right)=\frac{1}{N-k+1} \sum_{j=1}^{N\left(w_{1}\right)-k+1} h(k, j)
$$

where: $h(i, j)=1$, if a sub-sequence containing $i$ letters of word $w_{1}$ and

beginning from its $j$-th position in $w_{1}$, appears at least once in word $w_{2}$; otherwise: $h(i, j)=0$;

$h(i, j)=0$ also if $i>N\left(s_{2}\right)$ or $i>N\left(s_{1}\right)$;

$N=\max \left\{N\left(w_{1}\right), N\left(w_{2}\right)\right\}$ - the maximum of $N\left(w_{1}\right), N\left(w_{2}\right)$ - the

number of letters in words $w_{1}, w_{2}$, respectively;

$k$ denotes length of the considered string.

The function $\mu_{R W}$ can obviously be interpreted as fuzzy relation in terms of the fuzzy sets theory. This fuzzy relation is reflexive: $\mu_{R W}(w, w)=1$ for any word $w$; but in general it is not symmetrical. This inconvenience can be easily avoided by the use of minimum operation. Note that the human intuition is considered because the bigger is the difference in length of two words, the more different they are, and the more common letters are contained in two words, the more similar they are.

However, the value of the membership function contains no information on the sense or semantics of the arguments. In a natural way, the sentence comparison bases on word similarity measure and any two textual records which are sets of words (sentences or not) can be compared using formula (2).

Definition 2. The fuzzy relation on $S$ - the set of all sentences, is of the form $R S=\left\{\left(\left\langle s_{1}, s_{2}\right\rangle, \mu_{R W}\left(s_{1}, s_{2}\right)\right): s_{1}, s_{2} \in S\right\}$, with the membership function $\mu_{R S}$ : $S \times S \rightarrow[0,1]$ 


$$
\mu_{R S}\left(s_{1}, s_{2}\right)=\frac{1}{N} \sum_{i=1}^{N\left(s_{1}\right)} \max _{j \in\left\{1, \ldots, N\left(s_{2}\right)\right\}} \mu_{R W}\left(w_{i}, w_{j}\right),
$$

where: $w_{i}$ - the word number $i$ in the $s_{1}$ sentence, $w_{j}$ - the word number

$j$ in the $s_{2}$ sentence, $\mu_{R W}\left(w_{i}, w_{j}\right)$ - the value of the $\mu_{R W}$ function for the pair $\left(w_{i}, w_{j}\right)$, $N\left(s_{1}\right), N\left(s_{2}\right)$ - the number of words in sentences $s_{1}, s_{2}$, $N=\max \left\{N\left(s_{1}\right), N\left(s_{2}\right)\right\}$ - the number of words in the longer of the two sentences under comparison.

In the summary, we state that both methods, i.e. fuzzy concept based, and sequence kernels, used to find the similarity of words can be also applied to establish similarity of sentences or even whole documents. In the first method, some similarity function on the sentences or documents must be defined, cf.(2). In the second one, instead of letters, the alphabet should contain words or sentences. Both methods are non-sensitive to mistakes or other misshapen language constructions but standard preprocessing is recommended. Unfortunately, they do not use semantic information existing in the natural language. To increase the rate of comparison correctness a dictionary of synonyms should support the method applied.

\section{Summary}

At the beginning, RSS feed files were used only for the news sites. Now, with thousands of RSS-enabled sites, this format has become more popular, perhaps the most widely seen kind of XML. RSS-feed is easy to use and well optimized to retrieve the news from the source sites. That is why it is useful to publish the news in two formats; firstly HTML dedicated to visual presentation and secondly XML-based format which is more useful for retrieving-tools. The RSS format seems to be a good proposition for simple extracting textual records which play the same role in different documents and therefore needs to be evaluated on their mutual similarity or similarity to certain pattern. For human-similar automatic evaluation of document relevance more sophisticated methods for text comparison than simple classical identity relation must be applied. Comparison of longer textual documents (words, sentences, abstracts, full documents, etc.) becomes a key problem. Examples of two methods based on quantitative measures were presented. Because of their character, they should be integrated with other approaches and applied off-line, eg. as an intelligent agent module.

\section{References}

1. H. Bandemer and S. Gottwald. Fuzzy sets, fuzzy logic, fuzzy methods with applications. John Wiley and Sons, 1995.

2. N. Canccedda, E. Gaussier, C. Goutte, and J. M. Renders. Word-sequence kernels. Journal of Machine Learning Research, 3:pp. 1059-1082, 2003. 
3. L. Kangchan, M. Jaehong, and P. Kishik. A design and implementation of xmlbased mediation framework(xmf)for integration of internet information resources*. In Proceedings of the 35th Hawaii International Conference on System Sciences, New York, 2002. Association for Computing Machinery.

4. H. Lodhi, N. Cristianini, J Shave-Taylor, and C. Watkins. Text classification using string kernel. Advances in Neural Information Processing System, MIT Press, 13, 2001.

5. H. Lodhi, C. Sanders, J. Shave-Taylor, N. Cristianini, and C. Watkins. Text classification using string kernels. Journal of Machine Learning Research, 2:419-444, 2002.

6. E.T. Mueller. Machine-understandable news for e-commerce andweb applications. In CSREA Press, editor, Proceedings of the 2001 International Conference on Artificial Intelligence, pages 1113-1119, New York, 2001. Association for Computing Machinery.

7. A. Niewiadomski. Appliance of fuzzy relations for text documents comparing. In Proceedings of the 5th Conference on Neural Networks and Soft Computing, pages 347-352, Zakopane, Poland, 2000.

8. A. Niewiadomski, P. Kryger, and P.S. Szczepaniak. Fuzzy comparison of strings in faq answering. In BIS 2004. Proceedings of 7th International Conference on Business Information Systems, pages 355-362, Poznan, Poland, 2004. W.Abramowicz (Ed.), Wydawnictwo Akademii Ekonomicznej.

9. P.S. Szczepaniak and A. Niewiadomski. Clustering of documents on the basis of text fuzzy similarity. In Abramowicz W. (Ed.), editor, Knowledge-based information retrieval and filtering from the Web, pages 219-230. Kluwer Academic Publ, London, Boston, New York, Dordrecht, 2003.

10. P.S Szczepaniak and A. Niewiadomski. Internet search based on text intuitionistic fuzzy similarity. In P.S. Szczepaniak, J. Segovia, J. Kacprzyk, and L. Zadeh, editors, Physica-Verlag, A., Intelligent Exploration of the Web. Springer $\ddot{i}+\frac{1}{2}$ Verlag Company,, Heidelberg, New York, 2003.

11. K. Wegrzyn-Wolska. Etude et realisation d'un meta-indexeur pour la recherche sur le Web de documents produits par l'administration francaise. PhD thesis, Ecoles Superieures de Mines de Paris, DEC 2001.

12. K. Wegrzyn-Wolska. Fim-metaindexer a meta-search engine purpose-bilt for the french civil service and the statistical classification and evaluation of the interrogated search engines. In The Second International Workshop on Web-based Support Systems, In IEEE WIC ACM WI/AT'04, pages 163-170, 2004.

13. K. Wegrzyn-Wolska. Le document numerique: une etoile filante dans l'espace documentaire. Colloque EBSI-ENSSIB; Montreal 2004, 2004. 